\title{
Modulating the Neutron Flux from a Mirror Neutron Source
}

D. D. Ryutov

September 2, 2011

Internation Workshop "Fusion for Neutrons and Sub-Critical Nuclear Fission"

Varenna, Italy

September 12, 2011 through September 15, 2011 
This document was prepared as an account of work sponsored by an agency of the United States government. Neither the United States government nor Lawrence Livermore National Security, LLC, nor any of their employees makes any warranty, expressed or implied, or assumes any legal liability or responsibility for the accuracy, completeness, or usefulness of any information, apparatus, product, or process disclosed, or represents that its use would not infringe privately owned rights. Reference herein to any specific commercial product, process, or service by trade name, trademark, manufacturer, or otherwise does not necessarily constitute or imply its endorsement, recommendation, or favoring by the United States government or Lawrence Livermore National Security, LLC. The views and opinions of authors expressed herein do not necessarily state or reflect those of the United States government or Lawrence Livermore National Security, LLC, and shall not be used for advertising or product endorsement purposes. 


\title{
LLNL-PROC-497401 Modulating the Neutron Flux from a Mirror Neutron Source
}

\author{
D.D. Ryutov \\ Lawrence Livermore National Laboratory, Livermore, CA 94551, USA
}

\begin{abstract}
A 14-MeV neutron source based on a Gas-Dynamic Trap will provide a high flux of $14 \mathrm{MeV}$ neutrons for fusion materials and sub-component testing. In addition to its main goal, the source has potential applications in condensed matter physics and biophysics. In this report, the author considers adding one more capability to the GDT-based neutron source, the modulation of the neutron flux with a desired frequency. The modulation may be an enabling tool for the assessment of the role of non-steadystate effects in fusion devices as well as for high-precision, low-signal basic science experiments favoring the use of the synchronous detection technique. A conclusion is drawn that modulation frequency of up to $1 \mathrm{kHz}$ and modulation amplitude of a few percent is achievable. Limitations on the amplitude of modulations at higher frequencies are discussed.
\end{abstract}

Keywords: Materials tests; fusion neutrons; gas-dynamic trap

PACS: $52.55 . J d ; 28.52 . \mathrm{Fa}$

\section{INTRODUCTION}

A 14-MeV neutron source based on the Gas-Dynamic Trap (e.g., [1] and references therein) will provide a high flux of $14 \mathrm{MeV}$ neutrons for fusion materials and sub-component testing. Its design is well supported by the existing experimental data from the on-going mirror experiment at Novosibirsk [2, 3]. The flux of $2 \mathrm{MW} / \mathrm{m}^{2}$ of 14 $\mathrm{MeV}$ neutrons would allow performing the life-time tests of critical components of the first wall and blanket. In addition to its main goal, the source has potential applications in condensed matter physics, chemistry and biophysics. In this report, the author considers adding one more capability to the GDT-based neutron source, the modulation of the neutron flux with a desired frequency in the range from $\sim 1 \mathrm{~Hz}$ to $\sim 1 \mathrm{kHz}$.

The burning fusion plasma in ITER and future tokamak-based fusion reactors will not be in a perfectly steady state, due to the plasma fluctuations associated with a high toroidal current. In particular, so-called ELM (Edge Localized Mode) events lead to quasi-periodic dumping of a few percent of the plasma stored energy and occur with a tunable frequency of $30 \mathrm{~Hz}-1 \mathrm{kHz}[4,5]$. This will certainly affect the neutron production and lead to fluctuations of the neutron output. Variation of the neutron flux could be also caused by global plasma displacements. There can be other sources of the "flickering" of the burning plasma, in particular, thermal instabilities of a burning plasma [6].

The presence of the time-variations of the neutron flux will affect the performance and longevity of the blanket and plasma-facing components. On the other hand, the characteristic frequency and amplitude of the neutron flux variations will be devicespecific and cannot be predicted at present with a full certainty. It is therefore desirable 
to have a neutron source with a capability of generating the modulated neutron flux with adjustable amplitudes and frequencies.

The modulation may be also an enabling tool for high-precision experiments favoring the use of the synchronous detection technique. In the area of a solid-state physics, a response of such properties as electrical resistivity and thermal conductivity to periodic variation of the neutron flux may help in finding the characteristic relaxation times and identifying underlying mechanisms; similarly, the modulation would be useful in experiments on radiation-induced chemical effects.

We do not consider the rotating mechanical modulators periodically disrupting the flow of fusion neutrons: it is hard to imagine such modulators reliably working in a strong magnetic field in the vicinity of a neutron-producing plasma. They may probably be of some use in dedicated beamlines, well outside the blanket and the magnetic system, although the $14 \mathrm{MeV}$ spectrum will be very strongly dilated there.

\section{THE SOURCE CHARACTERISTICS}

The general configuration of the neutron source that we will be considering is shown in Fig. 1. Detailed description of various aspects of the source operation can be found in Refs. [1], [7] and [8]. When using these references, one has to remember that, during the twenty-five years since this concept of the source had been proposed, a large number of versions of the source had been assessed, with a broad range of possible operation parameters. In this note, we use the set of parameters for the medium-scale source, Table 1. This source would produce a $14 \mathrm{MeV}$ neutron flux of $\sim 1.5 \mathrm{MW} / \mathrm{m}^{2}$ on the surface of the test zone. General schematic of such a neutron source is shown in Fig. 1

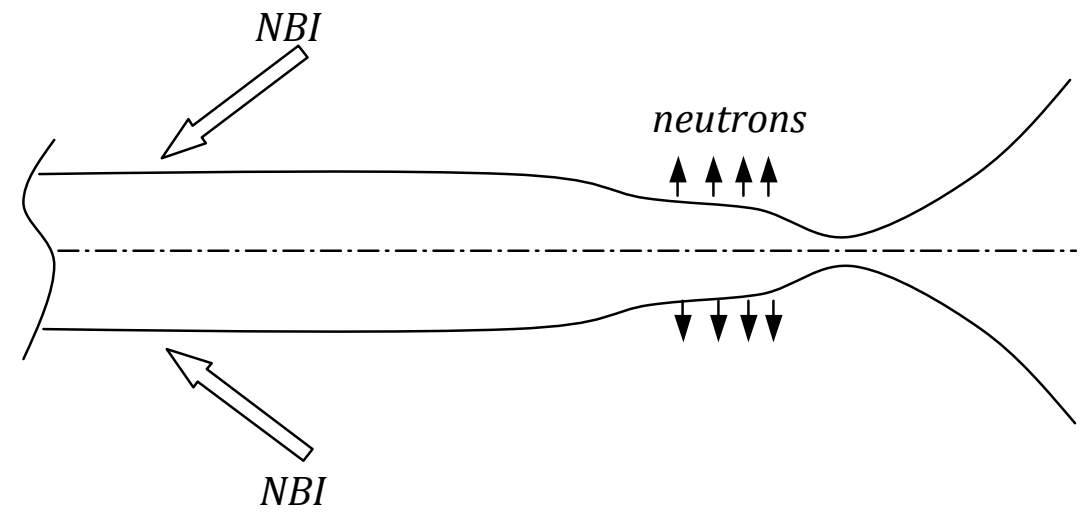

FIGURE 1. A general schematic of a neutron source based on the axisymmetric mirror with a skewed neutral beam injection (NBI). Solid lines show a plasma boundary (and the corresponding magnetic surface). Only a right part of the device is shown. The neutral beam injection occurs in the central section. The "shelve" on the magnetic surface corresponds to a reflection zone of the sloshing ions: here the neutron flux is maximum, and the test zones (one on each end) are situated. The figure is not to scale: the radial dimensions are exaggerated.

A high neutron flux is reached by injecting atomic beams into a target plasma with a relatively low electron temperature, at an angle to the magnetic axis. The slowingdown time of the energetic ions is significantly shorter than their angular scattering time (Table 2). Therefore, the turning points of their axial bouncing are approximately the 
same for all the ions, meaning a significant density (and the neutron production) increase at the turning point. One can "stretch" the high-density zones by making a "shelf" on the magnetic field axial dependence (Fig. 1). The achievable length of the test zones (there is a second one, at the left side of the device) is limited by the NBI power. For the trapped power of $\sim 20 \mathrm{MW}$ the length can be $\sim 1 \mathrm{~m}$.

\section{Table 1 Plasma parameters of the neutron source}

$\begin{array}{ll}\text { Mirror-to-mirror length } & L=12 \mathrm{~m} \\ \text { Plasma radius in the midplane } & a_{0}=20 \mathrm{~cm} \\ \text { Magnetic field in the midplane } & B_{0}=2 \mathrm{~T} \\ \text { Magnetic field in the test zones } & B_{t}=4 \mathrm{~T} \\ \text { Magnetic field in the mirrors } & B_{M}=16 \mathrm{~T} \\ \text { Electron density in the test zone } & n_{e t}=10^{14} \mathrm{~cm}^{-3} \\ \text { Electron temperature } & T_{e}=1 \mathrm{keV} \\ \text { Ion injection energy } & W_{i}=80 \mathrm{keV}\end{array}$

Operating in this, relatively energy-inefficient regime is beneficial for several reasons. First, in terms of the dimensionless parameters, it does not deviate significantly from those already produced on the GDT device [2, 9]; it is micro- and MHD stable in the framework of an axisymmetric geometry (Cf. a recent review [10]). Second, the rapid slowing down of the injected ions means that they have a small angular spread and are mirror-reflected in approximately the same field; this means that the neutron production is well localized so that the rest of the device experiences a relatively small neutron flux, and the facility has a long life-time.

\section{Table 2 Derived parameters of the neutron source}

$\begin{array}{ll}\text { Ion slowing-down time } & \tau_{\mathrm{S}}=0.08 \mathrm{~s} \\ \text { Ion scattering time } & \tau_{\mathrm{ii}}=2 \mathrm{~s} \\ \begin{array}{l}\text { Ion bounce time } L / \mathrm{v}_{\mathrm{i}} \\ \begin{array}{l}\text { Ion gyro-radius near the } \\ \text { turning points }\end{array}\end{array} & \tau_{\mathrm{b}}=3 \times 10^{-6} \mathrm{~s} \\ & \rho_{\mathrm{i}}=1.5 \mathrm{~cm}\end{array}$

\section{MODULATING THE NEUTRON FLUX}

The number $q$ of neutrons produced per unit length of the test zone per unit time can be presented as

$$
q=\pi a_{T}^{2} n^{2} \frac{\langle\sigma \mathrm{v}\rangle}{4}
$$

where $\langle\sigma \mathrm{v}\rangle$ is a DT reactivity, and we assume an equi-component mixture of D and T. Conceptually, the simplest way to produce a modulation would be to modulate the parameters of the neutral beam injectors (NBI), in particular, the injected current and/or the energy (the latter affects the fusion reactivity). However, this may be hard to do, because the NBI are the subtlest engineering sub-system of the whole facility, not 
amenable to any interference with its steady-state operation mode. In particular, its longevity may be adversely affected by the variations of the temperature of the accelerating grids and enhanced sputtering caused by the modulation. Note that a technique based on the modulation of the injection energy with a subsequent time-offlight bunching of the injected ions has been considered earlier in Ref. [8]; the conclusion was drawn that the modulation of a frequency of a few tens of kilohertz is possible with this approach.

On the other hand, if one does not rely on a complex technique of a programmed sweeping of the injection energy, just modulates the injection current, one encounters limitations on the achievable modulation frequency imposed by the basic properties of the plasma in the source. Consider, for example, that we are varying the injection current, thereby aiming at modulation of the density of the fast ions. The temporal dependence of the density can be represented by an equation: $\dot{n}=S-n / \tau$, where $S$ is the number of particles produced by NBI per unit volume per unit time, and $\tau$ is the ion lifetime in the device. In the GDT-based neutron source the latter is approximately equal to the slowing-down time (Table 2). Assume that the source $S$ is modulated with respect to its steady-state level $S_{0}: S=S_{0}\left(1+\varepsilon e^{-i \omega t}\right)$, where $\varepsilon$ is assumed to be small. The density modulation then is

$$
\frac{\left|\delta n_{i}\right|}{n_{i 0}}=\varepsilon \frac{1}{\sqrt{1+(\omega \tau)^{2}}},
$$

where we assume that $\varepsilon$ is positive. Equation (2) shows that, for the high-frequency modulation, with $\omega>>1 / \tau \sim 8 s^{-1}$, even quite a deep modulation of the source strength would yield only a minor variation of the neutron flux. Note that the modulation period for $\omega \sim 1 / \tau$ would be quite long, $2 \pi / \omega \sim 0.8 s$.

We therefore consider a different approach based on the fact that the scattering time $\tau_{\mathrm{ii}}$ of fast ions is much longer than their slowing-down time $\tau_{\mathrm{s}}$. This is a property that allows a concentration of the neutron production in a well-defined region of the source, see, e.g., Refs. [1, 2, 3, 7]. It proves to be beneficial for controlled periodical axial displacement of a right boundary of a high-flux zone, so that the neutron production at the right end of the "shelf" decreases to a small value and then comes back (we are referring here to the right-hand test zone shown in Fig. 1).

The ions that are just trapped from the neutral beams on the uniform part of the facility have all the same pitch angle and energy. In the velocity space (related to the midplane) they are situated at the point $\mathrm{v}_{\|}=\mathrm{v}_{0} \cos \vartheta_{0}, \mathrm{v}_{\perp}=\mathrm{v}_{0} \sin \vartheta_{0}$, where the subscript " 0 " corresponds to the just injected ions. We assume that the initial injection angle is 45 degrees. The ions slow down much faster than scatter; by the time when their energy drops to a half of the injection energy, they scatter by a small angle $\Delta \vartheta<<1$ around the initial angle $\vartheta_{0}$,

$$
\Delta \vartheta \sim \sqrt{\tau_{S} / \tau_{i i}} \sim 0.2
$$

The shape of the area occupied by the slowing-down ions in the velocity space is shown in Fig. 2. As the ions with $\mathrm{W}_{0} / 2<\mathrm{W}<\mathrm{W}_{0}$ provide the most significant contribution to the neutron flux in the test zone, we concentrate on their behavior. The ions that are scattered to the smaller angles with respect to $\vartheta_{0}$ penetrate to higher magnetic fields, i.e., deeper into the test zone. Their turning point is situated at the magnetic field that is 
higher than the field corresponding to the initial injection angle $\vartheta_{0}$. This field will be different by some $\Delta B$ from the field $B_{T}$ (Table 1) that corresponds to the 45 -degree pitch angle:

$$
\frac{\Delta B}{B_{T}}=2 \Delta \vartheta \cot \vartheta_{0}
$$

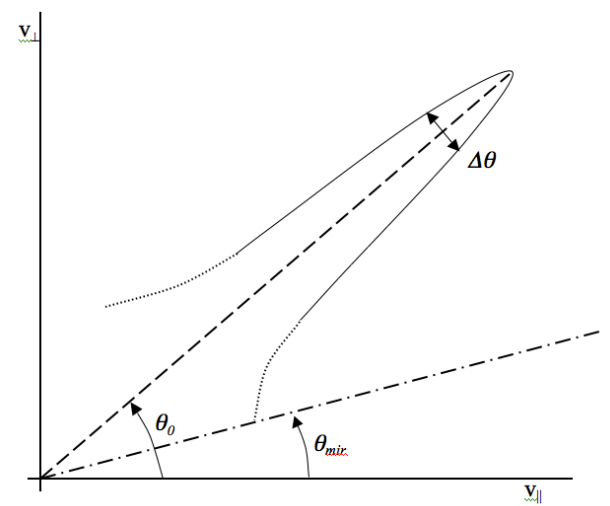

FIGURE 2. The slowing-down ions occupy a narrow zone around the line $\vartheta=\vartheta_{0}$ in the velocity space. At low velocities (which are of little interest for the neutron generation), the occupied zone rapidly broadens (as is qualitatively shown by a dotted line) and reaches a loss-cone boundary (a dash-dotted line).

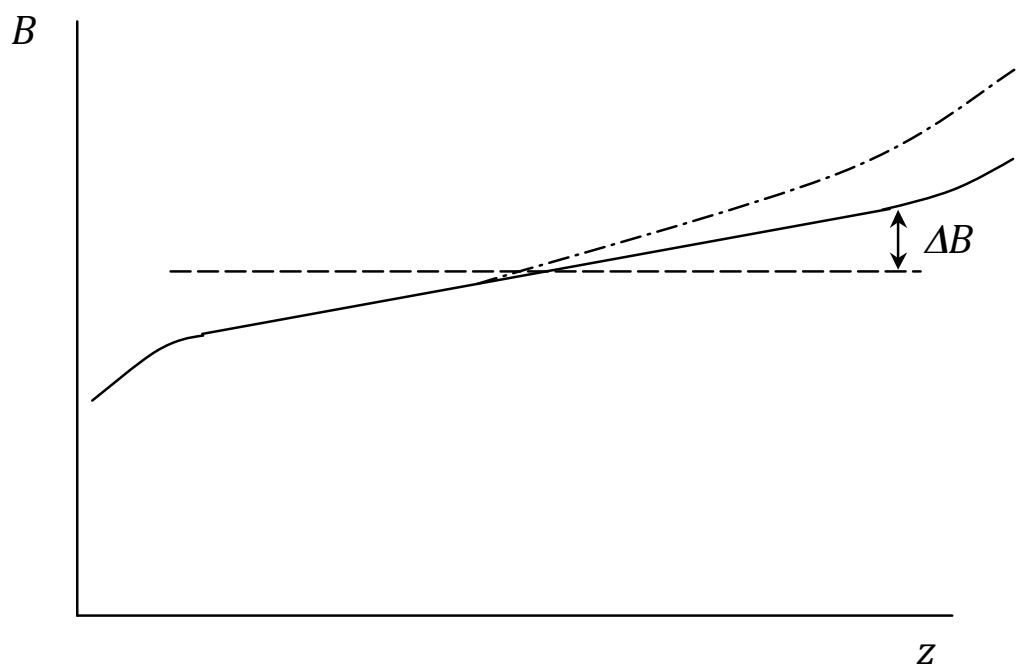

FIGURE 3. Axial dependence of the magnetic field in the "shelf" zone. The dashed horizontal line corresponds to the field $B_{t}$. The fast ions would not penetrate to the right end of the shelf if $\Delta B$ exceeds the value defined by Eq. (4). The length of the "shelf" is approximately $1 \mathrm{~m}$. The modulating coil can be situated $20-30 \mathrm{~cm}$ to the right of the right end of the "shelf", where the magnetic field is already $20-30 \%$ higher than the field at the end of the shelf. The resulting field distribution at the maximum of the cycle is shown in a dash-dotted line. At the minimum of the cycle, the field is as shown by the solid line.

This can be used for creating the neutron flux modulation. The magnetic field in the "shelf" (Fig. 1) should be made slightly non-uniform, as shown in Fig. 3, with the field on the right end being higher than $B_{T}$ by the value defined by Eq. (4). Then, the further increase of the field here would cut off the high-energy ions completely and lead to reduction of the neutron production by an order one, as only significantly slowed-down 
(and scattered) ions will be able to reach this point. To reach a 50\% modulation, one needs to modulate the magnetic field by $\sim 20 \%$. In other words, for the example presented in Table 1, the magnetic field has to be modulated by $\pm 4 \mathrm{kG}$. This is quite difficult if not impossible. In addition, the variation of the field should occur inside the superconducting coils, this further complicating the task.

On the other hand, if the field is modulated at an amplitude $\delta B<\Delta B$, then the modulation of the source strength will be of the order of $\delta B / \Delta B$. Therefore, if the modulation of $5 \%$ is needed, the magnetic field would have to be modulated by $\pm 200 \mathrm{G}$, which is a much easier task. The modulation frequency up to several kilohertz is technically feasible, although not simple.

Note that the modulating coils can be situated beyond the ion turning points (closer to the mirrors): they would create significant modulation at the axial distance comparable to their radius. Therefore, they are not necessarily interfering with the use of the test zone and are not necessarily exposed to the high neutron flux.

The smaller the modulation amplitude, the smaller is the phase volume affected by the modulation, the faster this volume is refilled via the ion scattering, and the higher modulation frequency is achievable. For the $1 \%$ modulation, a frequency of $1 \mathrm{kHz}$ is easily achievable.

\section{MODULATING THE NEUTRON FLUX AT A CONSTANT MAGNETIC FIELD}

In this section, we consider a possibility of moving the ion reflection points by modulating the electron temperature. The confinement time $\tau_{\text {cold }}$ of the colder plasma component is significantly shorter than the life-time of the hot ions: the colder plasma is heated by the slowing-down fast ions, at a rate $\sim n_{i} W_{i} / \tau_{S}$ per unit volume and lost via cold plasma outflow from the mirrors at a rate $\sim n_{\text {cold }} T_{e} / \tau_{\text {cold }}$. Here $n_{i}$ is the hot ion density and it is assumed (in agreement with more detailed calculations) that cold ions have a temperature not exceeding the electron temperature. For the parameters of the GDT experiment [2] and the related neutron source design, one has $n_{\text {cold }} \sim n_{i}$, this meaning that $\tau_{\text {cold }} \sim\left(T_{e} / W_{i}\right) \tau_{s} \sim 0.001 s$. By introducing the modulated auxiliary heating source, one can therefore modulate the electron temperature at frequencies up to $1 \mathrm{kHz}$. For the auxiliary heating, one can use, e.g., electron cyclotron heating in the zone near the mirrors, where the magnetic field is high. An alternative way of modulating $T_{e}$ could be the modulation of the gas injection that fuels the cold plasma: increased gas influx, leads to decrease of the electron temperature and vice versa. The high-rep-rate pellet injection could be used for this purpose.

The change of the electron temperature affects the position of the ion turning points via the change of the ambipolar electrostatic potential. The peak density of the sloshing ions is reached in the middle of the "shelf." This means that for those of the ions whose turning point is situated to the right of the peak, the presence of the ambipolar field leads to shifting of the turning points towards the mirror. We assume that the potential drop in the peak region is $\sim T_{e} / e$, this leading to the shift $\Delta z$ of the turning point by the distance $\Delta z \sim l\left(T_{e} / W_{i}\right)$. This is a modest displacement sufficient to relative 
change the neutron flux by the amount of $\Delta z / l \sim 0.015$. The advantage of this technique is that it does not require introducing additional magnetic field coils. All the manipulations would be provided either by the modulation of the gas injection, or the periodically-varying ECH power.

\section{DISCUSSION}

We have identified two techniques allowing for the controlled modulation of the neutron flux from the GDT-based neutron source. These techniques do not interfere with the operation of the neutral beam injectors. Moreover, the neutron flux in the major part of the test zones can be kept to a high accuracy constant, as the moving of the turning points occurs only in a limited region at the outer part of the test zones.

A few percent variation of the neutron flux at a frequency approaching $1 \mathrm{kHz}$ can be produced by the axial dither of the high-neutron-flux zone. The dither may be driven by the modulation of the magnetic field at a small section of a device, at the amplitude of $100-200 \mathrm{G}$. The modulating coils would be situated outside the highest flux region, this making them less vulnerable to neutron irradiation. Conceptually more attractive technique is based on the modulation of the ambipolar electric field by modulating the electron temperature. It does not require installing any additional hardware near the test zone and can operate by modulating the gas injection rate near the mirror. Periodic injection of pellets, also outside the region accessible for the sloshing ions, is another possibility. Yet another one is a modulation of the electron heating by a cyclotron resonance, with antennas situated near the mirrors. A drawback of this technique is that it can hardly provide the modulation amplitude higher than $1 \%$, at least for the source parameters considered in this paper.

A number of issues remains for the future analysis. In particular, we have not accounted for radial variation of the magnetic field in the central part of the source. This variation is caused by the finite plasma pressure and leads to an axial spread of the turning points. This (unfavorable) effect can be reduced by the proper aiming of the neutral beams, such that the radial pressure profile would be almost flat. The finite angular width of the atomic beams also leads to some spreading of the turning points of the just trapped ions.

The usefulness of the controlled modulation depends on the level of natural fluctuations of the plasma parameters. Dedicated measurements of the fluctuation spectrum in the existing GDT device could be of a significant help. In general, the mirrors, if MHD stable, do not show any low-frequency, high-amplitude variation of the plasma parameters like ELMs in tokamaks, but higher-frequency variations are possible. Spatial averaging of the short-wave-length fluctuations seems to be important in this regard.

\section{ACKNOWLEDGMENT}

This work was performed under the auspices of the U.S. Department of Energy by LLNS LLC Lawrence Livermore National Laboratory under Contract DE-AC52$07 N A 27344$. 


\section{References}

1. D.D. Ryutov, D.E. Baldwin, E.B. Hooper, K.I. Thomassen. J. Fusion Energy, 17, 253 (1998).

2. A.A. Ivanov, A.D. Beklemishev, E.P. Kruglyakov, P.A. Bagryansky, A.A. Lizunov, V.V. Maximov, S.V. Murakhtin, V.V. Prikhodko. Fusion Science and Technology, 57, 320 (2010).

3. P. A. Bagryansky, A. V. Anikeev, A. D. Beklemishev, A. S. Donin, A. A. Ivanov, M. S. Korzhavina, Yu. V. Kovalenko, E. P. Kruglyakov, A. A. Lizunov, V. V. Maximov, S. V. Murakhtin, V. V. Prikhodko, E. I. Pinzhenin, A. N. Pushkareva, V. Ya. Savkin, K. V. Zaytsev. Fusion Sci. Technol., 59, \#1T, 31 (2011).

4. D.N. Hill. Journ. Nucl. Materials, 241-243, 182 (1997).

5. A. Loarte, G. Saibene, R. Sartori, D. Campbell, M. Becoulet, L. Horton, T. Eich, A. Herrmann, G. Matthews, N. Asakura, A. Chankin, A. Leonard, G. Porter, G. Federici, G. Janeschitz, M. Shimada and M. Sugihara. Plasma Phys. Contr. Fusion, 45, 1549 (2003).

6. Y.I. Kolesnichenko, S.N. Reznik, V.A. Yavorskij. "Nucl. Fusion,” 16, 105 (1976).

7. E.P. Kruglyakov. Fusion Technology, 35, 20 (1999).

8. U. Fischer, A. Moslang, A.A. Ivanov. Fusion Technology, 35, 160 (1999).

9. T.C. Simonen, Fusion Sci. Technol. 57, 305 (2010).

10.D.D. Ryutov, H.L. Berk, B.I. Cohen, A.W. Molvik, T.C. Simonen. Phys. Plasmas, 18, 092301 (2011).

11. K.V. Lotov, A.A. Ivanov. Plasma Phys. Contr. Fusion 42, 1077 (2000).

12. R. W. Moir, N. N. Martovetsky, A. W. Molvik, D. D. Ryutov, T. C. Simonen. These Proceedings. 